\title{
Design of Fluxgate Sensors for Different Applications from Geology to Medicine
}

\author{
Ugur Topal $^{1} \cdot$ Hava Can $^{1,2}$. Oguzhan Merdan Çelik ${ }^{3} \cdot$ Ahmet Narman $^{4} \cdot$ Murat Kamiş $^{1} \cdot$ Veysel Çıtak $^{1}$. \\ Demet Çakrak $^{2}$ - Hüseyin Sözeri ${ }^{1} \cdot$ Peter Svec $^{5}$
}

Received: 18 May 2018 / Accepted: 19 June 2018 / Published online: 30 June 2018

(C) Springer Science+Business Media, LLC, part of Springer Nature 2018

\begin{abstract}
In this study, we have shown that fluxgate sensors can be used for different application areas. Alternative sensor configurations have been developed for three different application areas. These are mainly rod- and ring-type fluxgate sensors. In the first study, a measurement system with the rod-type fluxgate sensor has been developed in order to monitor the local magnetic field distributions on a magnetic tape (e.g., credit card). With this system, the magnetic recording data on the credit card were measured and imaged successfully. The second and the third application areas, which are monitoring the influence of solar storms on the earth field and heartbeat analysis of mankind, were carried out by using a ring-core fluxgate sensor geometry.
\end{abstract}

Keywords Magnetic sensors $\cdot$ Fluxgate sensors $\cdot$ Heartbeat analysis $\cdot$ Magnetic tape

\section{Introduction}

Magnetic field sensors have been widely used in different areas of technology. It was reported that they can be used for the detection of unexploded ammunitions and chemical, biological, and nuclear wastes. Detection of naval mines and submarines, studies of geophysical anomalies, and detection of underground water and petrol sources are some examples of these application areas [1-3]. In the application areas mentioned above, as well as in many other application areas, the sensitivity is a crucial parameter for the use of magnetic sensors. Detection of very low magnetic fields is achieved using superconducting quantum interference devices (SQUID)

Hava Can

sidellaa@gmail.com

1 Magnetism Laboratory, TÜBITAK-UME, P.K. 54, 41470, Kocaeli, Turkey

2 Department of Physics, Gebze Technical University, 41400, Kocaeli, Turkey

3 Department of E\&E Engineering, Gaziantep University, 27310, Gaziantep, Turkey

4 Department of E\&E Engineering, Bilkent University, 06800, Ankara, Turkey

5 Institute of Physics, Slovak Academy of Sciences, Dubravska cesta 9, 84511 Bratislava, Slovakia and fluxgate sensors. SQUID sensors have a very high sensitivity allowing for the detection of magnetic fields in the range of $10^{-10}$ to $10^{-15} \mathrm{~T}$. Despite that high sensitivity, SQUID sensors are less common than fluxgate sensors because of the need to use liquid helium and because they detect not the absolute value of magnetic field but the magnetic field change. The lowest magnetic field that can be detected using fluxgate sensors is of the order of $10^{-10} \mathrm{~T}$. It is known that fluxgate magnetometer is one of the most popular one in the magnetic sensor market. In addition to its usage in satellites as an attitude and orientation determination equipment, it has also applications in metal industry as a nondestructive testing method (NDT) and the traffic control systems. In the present work, we will show some useful applications of fluxgate sensors in different areas of technology. These applications are mainly fluctuations of the earth field by solar storms, local magnetic field distribution through a magnetic tape on the credit cards, and heartbeat analysis of the mankind.

\section{Results and Discussions}

\subsection{Monitoring the Influence of Solar Storms on the Earth Field}

The presence of Earth's magnetic field was announced to the whole world with a book written in 1600 by William M. 
Gilbert [4]. But the accurate and continuous measurement of the Earth's magnetic field corresponds to the 1840s. Karl F. Gauss who takes Gilbert's idea into account is the first scientist who established an observatory system and put forward theories [5]. The Earth is surrounded by a DC magnetic field created by a direct current that flows through the melted core of the crust of the earth. Direct current and magnetic field are maintained by a dynamo effect. The Earth's magnetic field is around 0.6 at poles and $0.3 \mathrm{G}$ at the equator. It is undergoing changes in magnitude over a time scale of thousands of years. These changes are not constant in time. Sometimes it can change very slowly; sometimes it can change extremely fast. The Earth's magnetic field shows a different distribution around the world due to diversity of its resources. This is why global changes in the Earth's magnetic field have different effects depending on geography. Besides, the solar wind provides flow of charged particles from the sun and interacts with the magnetosphere of the Earth. In the case of arrivals of the charged particle, solar storms, to the magnetosphere, its boundary is pushed towards the Earth. Currents set up on the boundary of the magnetosphere can cause an abrupt change on the Earth's magnetic field in different regions. Observatories established in different regions around the world examine the annual changes of magnetic field in their region and contribute to the determination of the Earth's magnetic field distribution by reporting the obtained scientific data. In order to obtain a healthy data, the accuracy of magnetic field measurements must be guaranteed. For this reason, the devices used in magnetic field measurements must be highly sensitive and robust against environmental parameters.

Many scientific research centers (NASA, ESA, etc.) in the world had announced that there would be severe solar storms in September and December 2014, which was expected to cause magnetic field changes up to $1000 \mathrm{nT}$. Therefore, magnetic field measurements were carried out during these periods in a magnetically clean environment in the UME building, and DC field changes in this area were recorded. Measurements were made by using a fluxgate magnetometer. A schematic view of the fluxgate magnetometer used in this study is shown in Fig. 1. The cores of the sensor have been prepared by using Metglas 2714A magnetic ribbons. This material with an atomic composition of $\mathrm{CoFeCrSiB}$ is chosen due to its wide availability from different commercial companies and extremely good magnetic and electrical properties. The excitation coils with 132 turns of copper wire having a diameter of $300 \mu \mathrm{m}$ were wounded around the ring core. The pick-up coils with 1840 turns of copper wire having a diameter of $100 \mu \mathrm{m}$ were wounded tightly. Measurement data of magnetic field were collected by an algorithm coded in LabVIEW programming language. Figures 2 and 3 show the results of the measurements made in months September and December, respectively. Observational data were compared

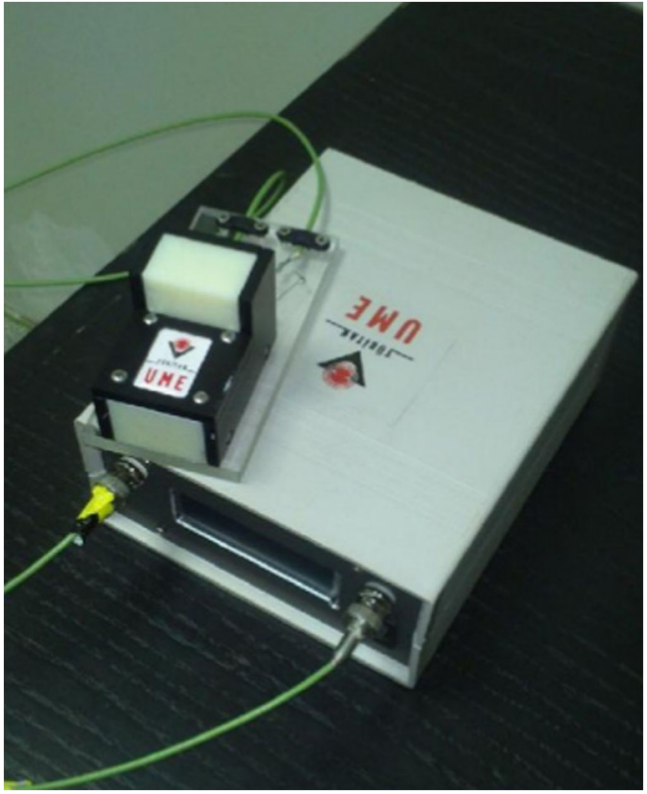

Fig. 1 Schematic view of the homemade ring-core fluxgate magnetometer

with the Swedish Institute of Space Physics (IRF) [6] and British Geological Survey (BGS) [7] data which provide real-time and open-access data. As seen from Fig. 2 that the daily change in the Earth's magnetic field is between 100 and $200 \mathrm{nT}$ in the UME building, between 50 and $400 \mathrm{nT}$ in IRF, and between 50 and $250 \mathrm{nT}$ in BGS. Besides, the daily change in the Earth's magnetic field in Fig. 3 is between 50 and $200 \mathrm{nT}$ in UME, between 200 and $450 \mathrm{nT}$ in IRF, and between 75 and $250 \mathrm{nT}$ in BGS. It is seen that the solar storm did not create an estimated effect on the Earth. However, it is clearly seen from the measurement results that there are different changes in different regions of the world. The changes in the magnetic field observations made in Sweden boundaries seem to be more with respect to Turkey and the UK. This difference may be due to Sweden's geographic location, which is closer to the North Pole.

\subsection{Local Magnetic Field Distribution Through the Magnetic Card}

Some of the typical features of the fluxgate sensors are high sensitivity, wide dynamic range, high resolution, low power consumption, and low cost [8-11]. The contribution of these features to its maintaining popularity is quite big. For this reason, the use of fluxgate sensors in different application areas is increasing day by day [12-17]. Finding an optimal design of fluxgate sensor suitable for nondestructive testing (NDT) and other small area applications requiring local field information were extensively investigated in our previous work [18]. With this study, i.e., minimizing the detectable sizes of flaws, cracks, and other small area field sources, a 
Fig. 2 Real-time monitoring of the Earth's magnetic field oscillations from 12 to 17 September

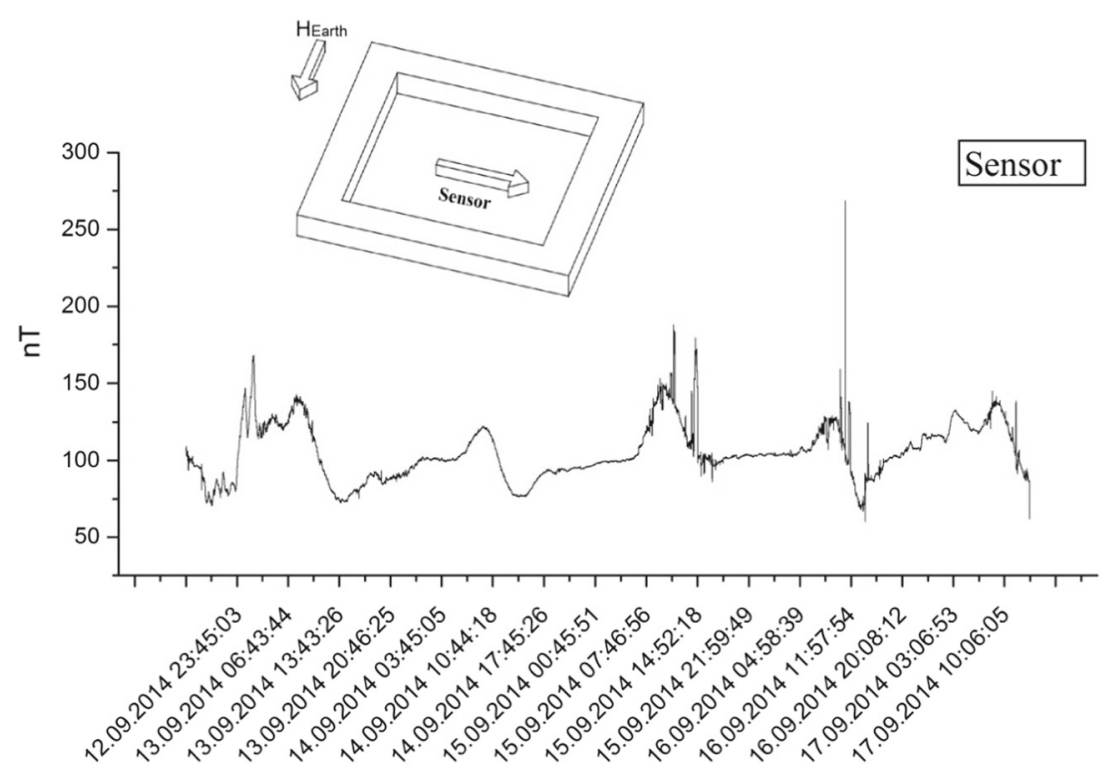

rod-type fluxgate sensor with a sensing area of $0.00785 \mathrm{~mm}^{2}$ has been fabricated. The winding system of the sensor comprises one hollow ceramic cylinder with an inner diameter of $200 \mu \mathrm{m}$, where the magnetic core is inserted, and two coils: the excitation coil and the pick-up coil. A threelayer pick-up coil with a length of $15 \mathrm{~mm}$, on which the harmonics of the fundamental frequency were induced, was wrapped tightly over the ceramic rod with a copper wire of $100 \mu \mathrm{m}$ in diameter. A single-layer excitation coil was wrapped over the pick-up coil with a copper wire of $200 \mu \mathrm{m}$ in diameter. The total length of the excitation coil was $20 \mathrm{~mm}$, and the winding changes direction at half of its length to minimize the zero offset value. Then, the magnetic core with a length of $19.6 \mathrm{~mm}$ was put into the coil system through the hole of the ceramic. Its position was adjusted according to the zero offset value. The length of the core was shorter than the excitation coil to ensure homogeneous magnetization of the core and longer than the pick-up coil in order to avoid noises coming from the bending of the magnetic field lines near the end of the cores. The raw magnetic core was obtained from Unitika Ltd and consists of cobalt, iron, silicon, and boron elements with nearly zero magnetostriction.

The local magnetic field distribution of the credit card with a magnetic tape was studied by using this rod-type fluxgate sensor. Measurement system and measurement results are shown in Fig. 4. The measurement system is computer controlled and consists of precise $x-y$ stage, lock-in amplifier,
Fig. 3 Real-time monitoring of the Earth's magnetic field oscillations from 21 to 29 December

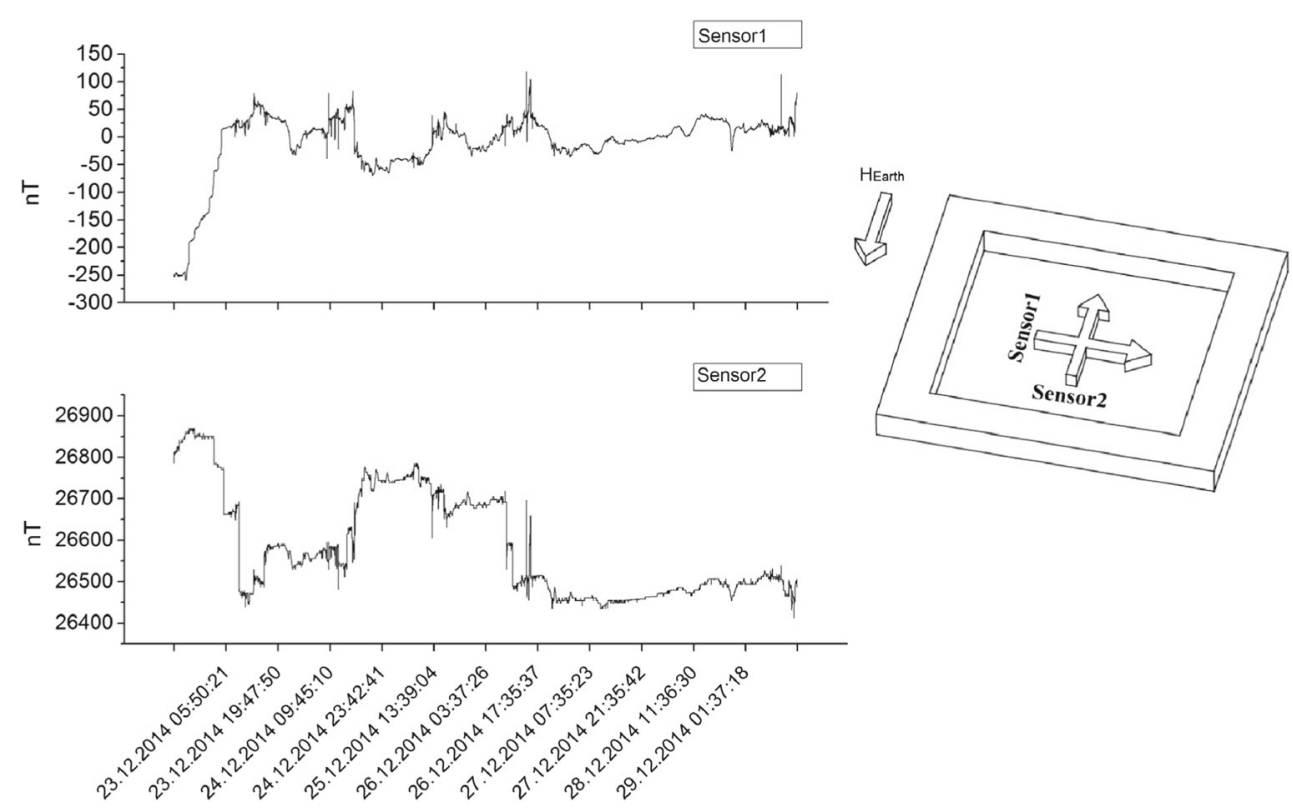


Fig. 4 Magnetic profile of a credit card obtained with rod-type fluxgate sensor. Notice that the image on the left and right sites were obtained with 50 and $10 \mu \mathrm{m}$ steps of the sensor. The numbers in the coordinate axes show the number of steps

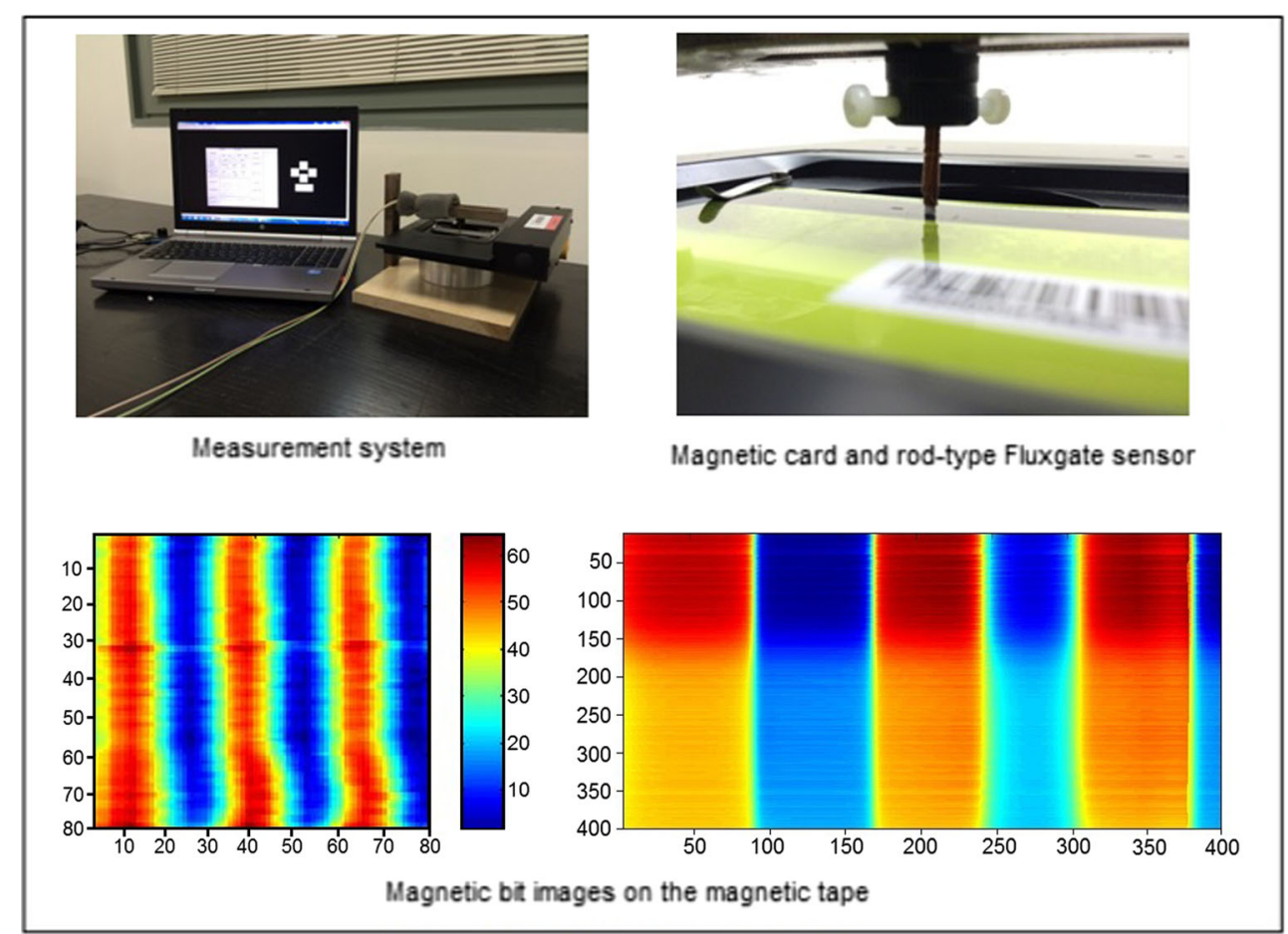

and the sensor. The automation of the system was done using the LabVIEW programming language. The $x-y$ stage used in the measurements has the ability to advance by $0.01 \mu \mathrm{m}$ steps. Measurements were made on the magnetic tape in a $4 \times 4 \mathrm{~mm}^{2}$ area. The obtained numerical data (voltage values read from the lock-in amplifier) were converted to the images seen in Fig. 4 by assigning color codes with the help of MATLAB programming language. There are two different bit images as seen in Fig. 4. The measurement on the left side was completed roughly in $2 \mathrm{~h}$ with $50 \mu \mathrm{m}$ steps (notice that the numbers in the coordinate axes show the number of steps). For this reason, the image obtained from this measurement is not clear. The measurement on the right side was completed in $10 \mathrm{~h}$ with $10 \mu \mathrm{m}$ steps. The edges of the bits in this measurement are sharper, and the resulting image is quite clear. As a result, it has been understood that the measurement quality can be determined according to the user's desire.

\subsection{Heartbeats Analysis with Fluxgate Sensor}

The magnetic field generated by the human heart was firstly measured in 1963 by G. Baule and R. McFee [19, 20]. The heart generates the largest magnetic field in the body. Human heart can generate a magnetic field of $100 \mathrm{pT}$. So, for detection of magnetic fields generated by the human heart, a quite precise and sensitive sensor operating at low frequencies is necessary. Generally, for the heart disorders not detected by electrocardiography and also for the examining the electrical activities of the brain, SQUID magnetometers are preferred, which sense the magnetic fields down to $10^{-15}$ T. However, these devices have high cost of manufacturing and servicing. Besides, the SQUID magnetometers cannot provide information about the direction of the magnetic field. For this reason, it is needed to use different methods and magnetometers to measure weak magnetic fields which could replace SQUID magnetometer. Recently developed fluxgate magnetometers which have very sensitive measurement capability, low cost, and easy to manufacture can be an alternative to SQUID magnetometers $[18,19]$.

In this study, the ring-core fluxgate sensor developed in ref. [21] was used for heartbeat analysis. The excitation coil of the sensor with toroidal windings of 180 turns was wound on the rings having a diameter of $20 \mathrm{~mm}$. The diameter of copper wire was $200 \mu \mathrm{m}$. The ring with excitation coil was put into the center of pick-up coil. The pick-up coil was wound from copper wire of having $100 \mu \mathrm{m}$ in diameter. The total numbers of turn was 2160 . Schematic view of the measurement setup is given in Fig. 5. The sensor was placed parallel to the heart with a gap of 1-2 mm. The measurements were done at sensor excitation frequencies of $12 \mathrm{kHz}$ for a period of $6-8 \mathrm{~s}$ by using SR830 Lock-in amplifier. In order to minimize the noise of measured signal and thus, to increase the $\mathrm{S} / \mathrm{N}$ ratio, a low noise preamplifier (SR560) was connected to the measurement system with 


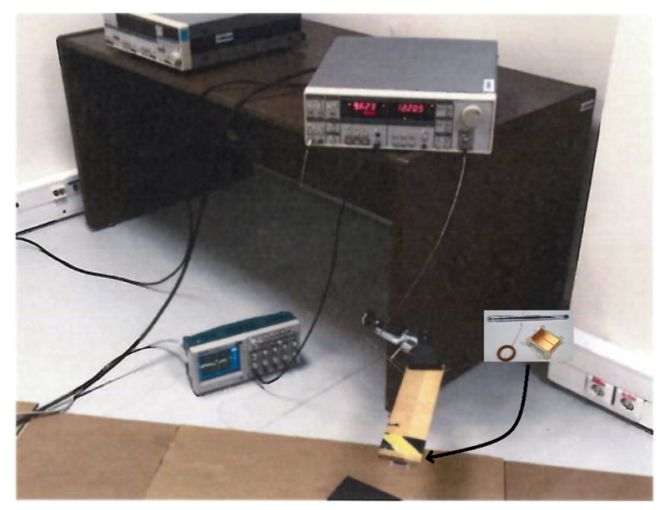

Fig. 5 Heartbeat measurement system

a gain factor of 100 . Both the sensitivity (1.9 MV/T) and the electrical noise $(0.9 \mathrm{pT} / \sqrt{ } \mathrm{Hz}$ at $1 \mathrm{~Hz})$ of the sensor is satisfactory to sense the fields larger than $10^{-11} \mathrm{~T}$, which is smaller than the typical field created by the heart $\left(\sim 10^{-10} \mathrm{~T}\right)$. It is important to note that the breath must be kept during the measurements to keep the distance between the heart and the sensor. The time constant of the lock-in amplifier was selected to be $30 \mathrm{~ms}$ to monitor all waves of the heartbeat since the heartbeat of a mankind at middle ages are known to be between 60 and $100 \mathrm{~min}^{-1}$, and the waves like QRS complex ends in $0.06-0.1 \mathrm{~s}$. It is known that the QRS complex, which is a name for the combination of three graphical deflections seen on ECG and corresponds to the depolarization of the right and left ventricles of the human heart, is the main spike seen on ECG. A Q wave is defined to be download deflection seen after $\mathrm{P}$ wave and $\mathrm{R}$ wave follows as an upward deflection. $\mathrm{S}$ wave is seen after $\mathrm{R}$ wave and seen as a downward deflection. Finally, $\mathrm{T}$ wave appears. From this knowledge, one can define these waves in the curve given in Fig. 6 and understand whether there are disease on certain part of the heart or not from the width and peak of these waves.

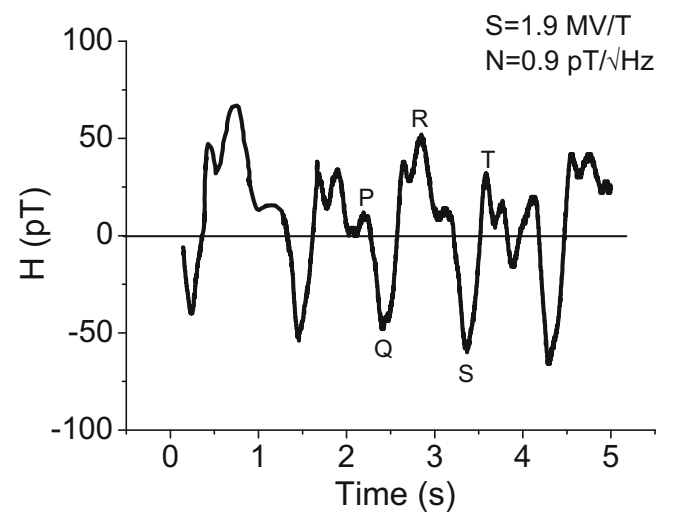

Fig. 6 Heartbeat analysis using the fluxgate sensor

\section{Conclusion}

In this study, we have shown the different applications of fluxgate sensors developed at different geometries. We could achieve to monitor the magnetic bits of a magnetic tape used in credit cards. It was also interesting to sense the heartbeats of the mankind. We suggest that one can even extract the magnetic map of the heart by using a fluxgate gradiometer on which many sensors are positioned at certain distances. Thus, any kind of disease on the heart can be detected from the magnetic data, like electrocardiogram (ECG).

Funding Information This work was supported by the Scientific and Technological Research Council of Turkey (TUBITAK), grant no. 113F192. Support by the joint SAS-TUBITAK project FX-GATES and VEGA $2 / 0082 / 17$ is gratefully acknowledged.

\section{References}

1. Peterson, J.C., Arredondo, R., Chao, T.-H., Friedman, G.L., LaBaw, C.: Sensor Technology Assessment for Ordinance and Explosive Waste Detection and Location. Revision B. Jet Propulsion Lab, Pasadena (1995)

2. Clem, T.R.: Progress in magnetic sensor technology for sea mine detection. Proc. SPIE-Int. Soc. Opt. Eng. 3079, 354-371 (1997)

3. Summey, D.C., Kekelis, G.J.: Fused airborne sensor technology. Detect. Remed. Technol. Mines Minelike Targets 2765, 226-232 (1996)

4. Gilbert, W.: Summary Article, the Hutchinson Dictionary of Scientific Biography. Helicon Publishing (2018)

5. Roberts, P.H., King, E.M.: On the genesis of the Earth's magnetism. Rep. Prog. Phys. 76(9), 1-55 (2013)

6. Swedish Institute of Space Physics Team: Geomagnetic development in real time. Institutet för rymdfysik (2014). [Online]. Available: http://www2.irf.se//Observatory/?link=Magnetometers. Accessed 10 Jan 2015

7. Geomagnetism Team: Lerwick Monthly Bulletins. British Geological Survey (2014). [Online]. Available: http://www.geomag. bgs.ac.uk/data_service/data/bulletins/ler.html. Accessed 01 Jan 2015

8. Ripka, P., Janosek, M.: Advances in magnetic field sensors. IEEE Sens. J. 10(6), 1108-1116 (2010)

9. Ripka, P.: Review of fluxgate sensors. Sens. Actuators: A Phys. 33(3), 129-141 (1992)

10. Janosek, M.: Parallel fluxgate magnetometers. Smart Sens. Meas. Instrum. 19, 41-61 (2017)

11. Moreland, C.: Fluxgate magnetometer. ReVision, pp. 1-9 (2002)

12. Sui, Y., Li, G., Wang, S., Lin, J.: Compact fluxgate magnetic fulltensor gradiometer with spherical feedback coil. Rev. Sci. Instrum. 85(1), 014701 (2014)

13. Xue, Q., Wang, R., Sun, F., Huang, Z.: Chaotic vibration analysis of the bottom rotating drill string. Shock. Vib. (2014)

14. Li, W., Wang, J.: Magnetic sensors for navigation applications: an overview. J. Navig. 67(2), 263-275 (2014)

15. Ciudad, D., Díaz-Michelena, M., Pérez, L., Aroca, C.: Small fluxgate magnetometers: development and future trends in Spain. Sensors 10(3), 1859-1870 (2010)

16. Kaluza, F., Grüger, A., Grüger, H.: New and future applications of fluxgate sensors 106 (2003) 
17. Herčík, D., Auster, H., Blum, J., Fornaçon, K., Fujimoto, M., Gebauer, K., Güttler, C., Hillenmaier, O., Hördt, A., Liebert, E., Matsuoka, A., Nomura, R., Richter, I., Stoll, B., Weiss, B.P., Glassmeier, K.: The MASCOT magnetometer. Space Sci. Rev. 208(1-4), 433-449 (2017)

18. Can, H., Svec, P., Tanrıseven, S., Bydzovsky, J., Birlikseven, C., Sözeri, H., Svec, P. Sr., Topal, U.: Optimizing the sensing performance of a single-rod fluxgate magnetometer using thin magnetic wires. Meas. Sci. Technol. 26(11), 115102 (2015)
19. McFee, R., Baule, G.M.: Research in electrocardiography and magnetocardiography. Proc. IEEE 60(3), 290-321 (1972)

20. Baule, G., McFee, R.: Theory of magnetic detection of the heart's electrical activity. J. Appl. Phys. 36(6), 2066-2073 (1965)

21. Can, H., Svec, P., Bydzovsky, J., Svec, P., Sözeri, H., Topal, U.: Systematic optimization of the sensing properties of ring-core fluxgate sensors with different core diameters and materials. Sens. Actuators A Phys. 255, 94-103 (2017) 